\section{La prevención olvidada: reemergencia del VIH en Chile}

\section{Forgotten prevention: the reemergence of HIV in Chile}

\section{Sr. Editor:}

En Chile pareciera que, al igual que los terremotos, los tomadores de decisiones no han podido predecir la reemergencia de la epidemia de VIH. Lo dicen desde hace años los organismos internacionales vinculados, lo advierten desde hace años las ONGs que trabajan en el tema, la evidencia científica lo deja claro, y sin embargo aquí estamos: nuestros datos preliminares muestran una prevalencia de VIH de 17,6\% en población homo-bisexual y otros hombres que tienen prácticas sexuales con otros hombres en la Región Metropolitana, la mayoría de ellos jóvenes (Proyecto VIBIMOS- FONDECYT 11140021) ${ }^{1}$. Una prevalencia que, considerando los aspectos epidemiológicos y socio-culturales actuales, probablemente en el norte del país se podría multiplicar por dos. Sucede que las cifras estratificadas, no los promedios mentirosos, muestran que hay subgrupos y territorios mucho más afectados $^{2}$.

El plan nacional de salud 2011-2020 tiene entre sus metas reducir la mortalidad por VIH/SIDA a 1,2 por 100.000 personas para el año 2020; en este plan no hay metas relacionadas con estrategias preventivas ${ }^{3}$. Según el último informe de $\mathrm{ONUSIDA}^{4}$, el número anual de nuevas infecciones por el VIH entre adultos en América Latina se ha mantenido estable desde 2010; sin embargo, las tendencias varían considerablemente entre los países. Mientras en Colombia, El Salvador, Nicaragua y Uruguay desde 2010 las nuevas infecciones han disminuido en más de $20 \%$, en Chile han aumentado en $34 \%$, liderando la Región de Las Américas, por encima de Guatemala (23\%), Costa Rica (16\%), Honduras (11\%) y Panama (9\%)

En términos de vigilancia del VIH e ITS, en el país hay que avanzar hacia la monitorización social, empezando por establecer el modelo de vigilancia de segunda generación ${ }^{5}$. Esto contribuirá a mirar el problema, no sólo en términos de riesgo de infección, sino también, en términos de determinación social de la enfermedad, considerando la especial vulnerabilidad de las mujeres y de la población joven, además de fenómenos sociales emergentes como la migración.

En terminos de prevención, hay que fomentar la prevención primaria y también la secundaria, detección oportuna de los nuevos casos para disminuir el $40 \%$ de retardo diagnóstico que actualmente hay en el país ${ }^{6}$. Para esto es necesario fortalecer el trabajo de las organizaciones de base comunitaria incorporando, por ejemplo, el testeo comunitario (check-points) como parte de la estrategia país, además de las actividades de acercamiento

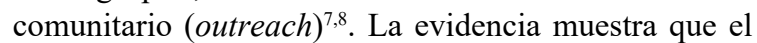
testeo de base comunitaria no sólo permite acceder a las poblaciones más vulnerables, sino también, identificar estrategias preventivas efectivas y la rápida vinculación de las personas al sistema sanitario (linkage to care) $)^{8,9}$.

En términos de control de la enfermedad, si bien el acceso al tratamiento anti-retroviral en Chile es universal, la baja adherencia es un problema que debe mirarse desde la perspectiva sociocultural y de género ${ }^{10,11}$. Esto impacta directamente sobre la calidad de vida de las personas con la infección por VIH, la mortalidad, la resistencia farmacológica y por su puesto sobre la prevención. En Chile se gasta más de $95 \%$ del presupuesto para VIH en tratamiento.

No es aceptable que un país con epidemia concentrada, que ha firmado compromisos internacionales en la materia, que tiene una Ley de SIDA y un programa nacional de prevención y control del VIH/SIDA, se haya olvidado de la importancia de la prevención en el control del VIH.

\section{Referencias bibliográficas}

1.- Stuardo V, y cols. Proyecto VIBIMOS- FONDECYT 11140021. Bio-behavioral surveillance for HIV/AIDS in hard-to-reach populations in the Metropolitan Region of Santiago, Chile.

2.- MINSAL, Dep. de Epidemiología. Evolución del VIH/SIDA Chile, 1984-2015.

3.- Ministerio de Salud de Chile. ESTRATEGIA NACIONAL DE SALUD Para el cumplimiento de los Objetivos Sanitarios de la Década 2011-2020. (2011). http:// www.ispch.cl/sites/default/files/documento/2011/12/ Metas2011-2020.pdf.

4.- Joint United Nations Programme on HIV/AIDS (UNAIDS). Ending AIDS Progress towards the 90-90-90 targets. GLOBAL AIDS UPDATE , 2017. http://www.unaids.org/ sites/default/files/media_asset/Global_AIDS_update_2017_ en.pdf.

5.- UNAIDS/WHO Working Group on Global HIV/AIDS and STI Surveillance. Guidelines for second generation HIV surveillance : an update : know your epidemic. WHO Guidelines. (2011). http://apps.who.int/iris/ bitstream/10665/85511/1/9789241505826_eng.pdf.

6.- Fast-Tracking Combination Prevention Unaids 2015 Reference towards reducing new HIV infections to fewer than 500000 By 2020. http://www.unaids.org/sites/default/ files/media_asset/20151019_JC2766_Fast-tracking_ combination_prevention.pdf.

7.- Fernàndez-Lopez L, Rifà B, Pujol F, Becerra J, Pérez M, Meroño M, et al. Impact of the introduction of rapid HIV testing in the voluntary counselling and testing sites network of Catalonia, Spain. Int J STD AIDS 2010; 21: 388-91.

8.- Qvist T, Cowan SA, Graugaard C, Helleberg M. High linkage to care in a community-based rapid HIV testing and counseling project among men who have sex with 


\section{Carta al Editor}

men in Copenhagen. Sex Transm Dis 2014; 41: 209-14. doi:10.1097/OLQ.0000000000000096

9.- Meulbroek M, Ditzel E, Saz J, Taboada H, Pérez F, Carrillo A, et al. BCN Checkpoint, a community-based centre for men who have sex with men in Barcelona, Catalonia, Spain, shows high efficiency in HIV detection and linkage to care. HIV Med 2013;14 Suppl 3: 25-8. doi:10.1111/hiv.12054

10.- Puskas C, Hogg R S. Unlocking adherence: is gender the key? lancet. HIV 2, e2-3 (2015).

11.- Stuardo Ávila V, Manríquez Urbina J M, Fajreldin Chuaqui V, Belmar Prieto J, Valenzuela Santibáñez V. Model of socio- cultural dimensions involved in adherence to antiretroviral therapy for HIV/AIDS in public health care centers in Chile. AIDS Care 2016; 28: 1441-7.

Valeria Stuardo

Programa de Epidemiología, Escuela de Salud Pública, Facultad de Medicina, Universidad de Chile.

Correspondencia: vstuardo@med.uchile.c 